\title{
Poststroke cognitive impairment and dementia: prevalence, diagnosis, and treatment
}

This article was published in the following Dove Press journal:

Degenerative Neurological and Neuromuscular Disease

5 February 2014

Number of times this article has been viewed

\section{Susanna Melkas \\ Hanna Jokinen \\ Marja Hietanen \\ Timo Erkinjuntti}

Department of Neurology, Helsinki University Central Hospital and Department of Neurological Sciences, University of Helsinki, Helsinki, Finland
Correspondence: Susanna Melkas Helsinki University Central Hospital, PO Box 372, Fl-00029 HUS, Helsinki, Finland

Email susanna.melkas@hus.fi

\begin{abstract}
Three aspects of poststroke cognitive impairment and dementia are discussed in this review: prevalence; diagnosis; and treatment. The aim is to increase awareness of poststroke cognitive impairment in order to further stimulate strategies to recognize the condition and to prevent its progression. Approximately two-thirds of all middle-aged and elderly stroke patients develop cognitive impairment, and one in three develops dementia. The standard reference for diagnosis is a detailed neuropsychological examination. Short screening tests have been used for both clinical and research purposes, but their sensitivity is limited and there is no consensus as to which test is the most appropriate. The treatment of poststroke cognitive impairment and dementia is based on effective treatment of vascular risk factors, including lifestyle modification when needed.
\end{abstract}

Keywords: cerebrovascular diseases, stroke, cognitive impairment, dementia

\section{Introduction}

Poststroke cognitive impairment (PSCI) is defined as failure in any cognitive domain after stroke: executive function; memory; language; visuospatial ability; visuoconstructional ability; or global cognitive function. Poststroke dementia (PSD) is defined as any dementia occurring after stroke: vascular dementia; Alzheimer's disease or other degenerative dementia; or mixed dementia. The concepts PSCI and PSD usually refer to conditions occurring after symptomatic strokes with corresponding ischemic findings upon neuroradiological imaging. Also, seemingly asymptomatic strokes - a common incidental finding in patients both with and without symptomatic strokes - add to the vascular burden of the brain.

Even though PSCI and PSD may not be purely vascular in origin, they can at least partly be regarded as categories of vascular cognitive impairment (VCI). ${ }^{1} \mathrm{VCI}$ has been proposed as an umbrella term to recognize the broad spectrum of cognitive and behavioral changes associated with vascular pathology. ${ }^{2}$ The most common causes of VCI are small-vessel disease, large-vessel disease, and strategic infarcts that may be either cortical or subcortical. ${ }^{3}$ VCI may also be the result of hypoperfusion, hemorrhage, and hereditary conditions (for example, cerebral autosomal dominant arteriopathy with subcortical strokes and leukoencephalopathy). All of these subtypes of VCI may appear poststroke.

The widening use of magnetic resonance imaging influences and may even exaggerate the estimate of the vascular burden behind cognitive impairment because vascular changes are more apparent than degenerative ones in neuroradiology. ${ }^{4}$ On the other hand, depending on their location, vascular lesions may be more expressive than 
they seem, disrupting interconnections between several distal regions. This applies, for example, to lesions in the basal ganglia and to the deep white matter, which damage the frontal-subcortical network and associate with executive dysfunction. ${ }^{5}$ The thalamus is another example of a strategic infarct location that may also cause amnestic dysfunction, in addition to executive defects. ${ }^{6}$

According to prevalence studies, ${ }^{7,8}$ the time of the first cognitive assessment varies from 7 days to 20 months after the index stroke. Assessment at 3 months poststroke has been widely adopted in research, which is in line with the common practice of assessing a patient's functional status at 3 months using the modified Rankin scale. ${ }^{9}$ The longitudinal follow-up times of PSCI and PSD cohorts extend to up to 25 years. $^{10}$

\section{Course and prognosis of poststroke cognitive disorders}

The major determinants of PSCI and PSD are advancing age, low education, and prestroke cognitive impairment or dependency. ${ }^{11}$ Standardized questionnaires such as the Informant Questionnaire on Cognitive Decline in the Elderly enable the assessment of prestroke cognitive status in order to differentiate new-onset PSCI from prestroke conditions. ${ }^{12,13}$ Prestroke dementia has been shown to associate with medial temporal lobe atrophy and family history. ${ }^{14}$ However, the exclusion of patients with prestroke cognitive impairment from a study does not necessarily exclude all patients with an Alzheimer-type pathology. ${ }^{15}$

A stroke seems to be able to initiate a vascular exacerbation that increases the likelihood of clinical dementia diagnosis in a patient with, for example, subclinical Alzheimer's disease. ${ }^{2}$ In the elderly in particular, incident ischemic cerebrovascular disease is related to the clinical expression of dementia associated with Alzheimer's disease pathology. ${ }^{16}$ It has been suggested that microangiopathy-induced leakage of apolipoprotein E might represent a pathogenetic link between cerebrovascular disease and Alzheimer's disease. ${ }^{17}$ A degenerative component seems to be important particularly in early-onset PSD, which refers to dementia that develops immediately after stroke. ${ }^{18}$

An important aspect in PSCI and PSD is the influence of a previous stroke. Some studies pinpoint multiple strokes as the central cause of the transition from PSCI to PSD, ${ }^{14}$ while others highlight the cumulative effect of white matter changes, hypertension, and amyloid deposits in the brain's parenchyma and blood vessels. ${ }^{19-21}$ As demonstrated in the multicenter European collaboration study, the Leukoaraiosis and Disability in the Elderly Study (LADIS), ${ }^{21}$ severe white matter changes predict a rapid decline in global functioning and more than double the risk of dependency during a 3-year follow-up. All three mechanisms are probably of importance and offer targets for preventive strategies: degenerative mechanisms; recurrent stroke; and the progression of smallvessel disease.

Cognitive impairment no dementia is a potentially reversible condition; approximately $16 \%-20 \%$ of elderly stroke patients with cognitive impairment improve during follow-up. ${ }^{22-24}$ However, cognitive impairment no dementia is associated with poor poststroke prognosis in terms of functional independence and survival. ${ }^{25}$ Deficits in executive functions, and visuospatial/constructional abilities in particular, have been associated with impaired mean survival from approximately 10 years to 6 years in a cohort of stroke patients aged $55-85$ years. $^{25}$

When the deficits are serious enough to meet the criteria of dementia, the prognosis becomes deleterious, not least because of the failing adherence to secondary prophylaxis and the increased risk of recurrent stroke. ${ }^{26}$ Several studies have shown that PSD is independently associated with increased poststroke mortality, after adjusting for vascular risk factors and stroke severity. ${ }^{12,13,27}$ It is still unclear to what extent the prognosis of PSD improves with the more intensive treatment of vascular risk factors, as compared with standard treatment. The emerging results of the Secondary Prevention of Small Subcortical Strokes trial will address this question, comparing intensive blood pressure control with standard blood pressure control and investigating the prevention of recurrent stroke and the reduction of cognitive decline as primary outcomes. $^{28}$

\section{Factors influencing prevalence and incidence}

It is acknowledged that approximately two-thirds of stroke patients develop any cognitive decline (ie, either cognitive impairment or dementia). ${ }^{11}$ One in three patients develops dementia, ${ }^{11,14}$ with the prevalence and incidence being highest within the first year after stroke. ${ }^{11,23}$ However, the prevalence of PSCI and PSD varies due to the heterogeneity of the studies. The heterogeneity is mainly explained by three factors: whether the study is hospital-based or populationbased; whether patients with prestroke dementia were included or excluded; and whether only first-ever or only recurrent strokes, or both, were included. ${ }^{14}$ The distribution of the anatomical location of stroke in the samples is also variable and influences the outcome. ${ }^{14}$ 
Other varying factors are the age of the study population, the timing of the cognitive assessment, and the differing exclusion criteria (for example, the presence of aphasia). As vascular dementia is the most important category of PSD, the diagnostic criteria used for vascular dementia greatly influence the exact prevalence rate. Several studies have shown that the prevalence rate of poststroke vascular dementia is lowest when the National Institute of Neurological Disorders and Stroke - Association Internationale pour la Recherche et l'Enseignement en Neurosciences (NINDSAIREN) criteria are used, and highest when the Diagnostic and Statistical Manual of Mental Disorders, 3rd Edition (DSM-III) and the International Classification of Diseases, 10th Edition, Neurological Adaptation (ICD-10-NA) criteria are applied. ${ }^{23,29}$ The NINDS-AIREN criteria require evidence of cerebrovascular disease in imaging, whereas the DSM-III and ICD-10-NA do not require such direct evidence. In most studies on poststroke cognitive decline, the diagnoses lack neuropathological verification, leading to some degree of uncertainty concerning the diagnoses. ${ }^{30}$

\section{The prevalence and incidence of PSD}

Leys et $\mathrm{al}^{11}$ showed in their review from 2005 that in community-based studies, the prevalence of PSD was approximately $30 \%$, whereas in hospital-based studies the prevalence ranged from $6 \%$ to more than $32 \%$. Incidence studies showed that stroke doubles the risk of dementia, and that this risk is at its highest within the first 6-12 months. Furthermore, the risk of delayed dementia, including presumed Alzheimer's disease, was also doubled after stroke. The follow-up times in the reviewed prevalence studies were up to 36 months.

The systematic review and meta-analysis by Pendlebury and Rothwell ${ }^{14}$ indicated that the PSD prevalence rates range from $7 \%-41 \%$ - the lower percentage appearing in population-based studies of first-ever stroke patients, and the higher in hospital-based studies of recurrent stroke in which prestroke dementia was included. Of patients suffering a first-ever stroke, 10\% soon developed new-onset dementia, and more than one in three suffered from dementia after a recurrent stroke. The follow-up in the hospital-based studies lasted predominantly up to 1 year, whereas in populationbased studies, the follow-up extended to up to 25 years.

\section{The prevalence of PSCI}

Most studies on PSCI have used brief screening measures in assessing global cognitive status - for example, the
Mini-Mental State Examination (MMSE) or the Abbreviated Mental Test ${ }^{31}$ - instead of a more detailed neuropsychological evaluation. Therefore, the full prevalence of multifaceted neuropsychological symptoms in stroke patients remains to be discovered in detail.

In the Helsinki Stroke Aging Memory ${ }^{32}$ cohort of patients aged 55-85 years, a comprehensive neuropsychological assessment was conducted 3 months after the index stroke. This revealed that $83 \%$ of the patients showed impairment in at least one cognitive domain and $50 \%$ were impaired in multiple ( $\geq 3$ ) domains 3 months poststroke. In cases with good clinical recovery (modified Rankin Scale $\leq 2$ ), the prevalence of any cognitive deficit was still 76\% (Hietanen et al, unpublished data, 2014). Only $29 \%$ of the total sample and $13 \%$ of the cases with good clinical recovery scored below 25 on the MMSE. The 12-year follow-up revealed that cognitive impairment, particularly in executive functions and visuospatial/constructional abilities, relates to poor survival. ${ }^{25}$

The Sydney Stroke Study ${ }^{15}$ analyzed the neuropsychological profile of VCI and vascular dementia in stroke patients, including patients with a history of a previous stroke. Two in five patients were diagnosed as having vascular mild cognitive impairment 3-6 months after the stroke, and one in five patients met the criteria for a diagnosis of dementia. The domains that were most affected in patients with cognitive impairment were abstraction, mental flexibility, information processing speed, and working memory.

In the South London Stroke Register, ${ }^{33}$ cognitive impairment was assessed with the Abbreviated Mental Test 3 months after the first-ever stroke and at annual follow-up visits for 14 years. The overall prevalence remained relatively stable: $22 \%$ at 3 months; $22 \%$ at 5 years; and, finally, $21 \%$ at 14 years. A significant, progressive trend in cognitive impairment was associated with small-vessel occlusion and lacunar infarction.

A recent review by Makin et $\mathrm{al}^{34}$ on cognitive impairment after lacunar stroke showed that approximately 30\% of patients develop cognitive impairment in the 4 years following a lacunar stroke, a proportion that is comparable to nonlacunar stroke. Accordingly, in the LADIS, ${ }^{35}$ the number of new lacunes during a 3-year follow-up was related to progressive cognitive impairment, which was apparent as a decline in executive functions, as well as in speed and motor control. Edwards et $\mathrm{al}^{36}$ found in their systematic review that cognitive impairment after lacunar stroke involves multiple cognitive domains. In an autopsy study, ${ }^{37}$ lacunes in thalamus, basal ganglia, and deep white matter in particular have been associated with cognitive decline. 


\section{Diagnosis}

The standard reference method for the assessment of cognition after stroke is the neuropsychological examination. In research, it is usually conducted 3 months after a stroke, but in clinical practice it follows earlier, from 1 week to 1 month after the stroke. However, a neuropsychological assessment is not always available for all stroke patients, and patients may be too disabled or fatigued to complete the test at the acute stage. Shorter screening tests for PSCI and PSD have been used both at clinics and for research purposes, but there is no consensus as to which test is the most appropriate. It is worthwhile to remember that screening tests have been developed for elderly patients in order to screen for a cognitive impairment that already meets the criteria for dementia. In less severe cases, the tests' lack of sensitivity is apparent.

The MMSE is widely used, but it is insensitive to mild cognitive impairment and executive function. ${ }^{38-40}$ The same applies for the Abbreviated Mental test, which is used in two variants: the Hodkinson Ten-Point abbreviated mental test and the shortened four-item test. ${ }^{30}$ The Montreal Cognitive Assessment (MoCA $)^{2}$ is more reliable since it also includes executive and attentional tasks, but its overall sensitivity for mild cognitive impairment is still restricted. The MoCA was originally proposed by the NINDS-Canadian Stroke Council harmonization standards for the detection of VCI for epidemiological research purposes. ${ }^{2} \mathrm{~A}$ recent study in a clinical setting indicated that the MoCA score at 5-9 days after stroke was independently associated with the occurrence of dementia at 6-9 months. ${ }^{41}$

The Cambridge Cognitive Examination (CAMCOG) and its shortened version, the Rotterdam-CAMCOG, are brief neuropsychological batteries that are used to detect a range of cognitive defects from mild cognitive impairment to dementia. ${ }^{42}$ Although originally designed to detect primary degenerative dementia, they have been used to detect PSCI. In a recent study in a population setting, ${ }^{43}$ the CAMCOG memory subscales predicted dementia accurately, but the nonmemory scales had lower accuracy.

In the setting of the LADIS, the Alzheimer's Disease Assessment Scale Cognitive Subscale and the Vascular Dementia Assessment Scale Cognitive Subscale (VADAS$\operatorname{cog})^{44}$ were compared to determine whether they differentiated between the three groups of white matter changes - mild, moderate, and severe. ${ }^{45}$ The VADAS-cog, and especially the additional tests measuring processing speed and executive functions, had a highly significant relationship with the white matter changes.
Lees et $\mathrm{al}^{46}$ recently compared the accuracy of several brief tests for the screening of cognitive impairment and delirium in acute stroke, using the MoCA as a reference standard for cognitive function, and the Confusion Assessment Method for delirium. ${ }^{46}$ The results suggest that the 4-A Tes $^{47}$ is a favorable choice for both delirium and cognitive screening. The 4-A Test is comprised of four components based on direct assessment and observations, enabling the differentiation between delirium and cognitive impairment. The authors conclude that informal assessment of cognition and delirium is not sufficient in acute stroke, but unfortunately the existing assessment instruments are not specifically designed for acute stroke. Further test accuracy assessments are needed to reach a consensus about which screening test would be optimal for the detection of cognitive impairment in stroke survivors.

\section{Treatment}

Due to the heterogeneity of PSCI and PSD, their potential treatment strategies range from preventing new strokes and preventing white matter changes, to treating hypertension as well as other underlying vascular risk factors, while preventing amyloid deposits. ${ }^{2,16}$ There are no treatment trials conducted specifically on patients with PSCI and PSD. In practice, the guidelines for the treatment of VCI and vascular dementia are applied, and in the case of Alzheimer's disease or mixed dementia, the target is to address the cholinergic deficit. The choice of antiplatelet drugs (aspirin, or aspirin plus dipyridamole, or clopidogrel) and anticoagulation is made according to the stroke prophylaxis guidelines. It is, however, important to note that beyond the acute phase treatment with alteplase, there are no other stroke treatments approved by the US Food and Drug Administration (FDA).

The management of vascular risk factors (hypertension, hyperlipidemia, diabetes, smoking, atrial fibrillation, and other arrhythmias) is essential, but little evidence exists on how this influences the course of cognitive impairment. The Perindopril Protection Against Recurrent Stroke Study (PROGRESS) and the PROGRESS magnetic resonance imaging substudy have emphasized the importance of antihypertensive treatment in prophylaxis against white matter changes, ${ }^{48,49}$ showing that the administration of an angiotensin-converting-enzyme inhibitor and a diuretic delayed the progression of white matter changes in patients with a clinical stroke.

Specific therapeutic trials on VCI have focused on vascular dementia, using NINDS-AIREN criteria. ${ }^{50}$ 
Modest cognitive improvement has been demonstrated with donepezil, galantamine, and memantine, but this is not reflected in global functioning. ${ }^{51-53}$ Neither VCI nor vascular dementia are official indications for any pharmacologic treatment in Europe (European Medicines Evaluation Agency) or in the US (FDA). In mixed dementia (Alzheimer's disease together with vascular components), galantamine seems to improve both cognition and global functioning. ${ }^{54}$

In PSCI and PSD, it is important to effectively treat associated conditions such as depression, delirium, sleep disorders, and pain. Several trials are currently being conducted regarding the treatment of poststroke depression. For example, fluoxetine has been shown to have an effect on both cognitive and motor recovery, ${ }^{55}$ and previously a small study on sertraline demonstrated benefits on executive function testing. ${ }^{56}$ As Alexopoulos et $\mathrm{al}^{57}$ have stated concerning the depression-executive dysfunction syndrome, executive dysfunction (but not other cognitive abnormalities) seems to influence the course of geriatric depression. ${ }^{57}$ Delirium during the first week after stroke is associated with an elevated risk of dementia at 3 months, ${ }^{58}$ and it must be treated promptly in order to minimize this risk. Poststroke fatigue also aggravates PSCI, ${ }^{34}$ and although it is challenging to treat, efforts can be made by, for example, managing sleep disorders.

Cochrane reviews have reviewed nonpharmacological interventions (mainly cognitive rehabilitation), but the conclusion is that more studies on this aspect are needed. ${ }^{50}$ Physical activity has been identified as a promoter of the structural integrity of the central nervous system, suggesting that physical activity counteracts age-related declines. ${ }^{59}$ A 4-year clinical trial testing an exercise intervention with cognitive function as a secondary outcome is expected to provide more information on the matter in the near future (The Lifestyle Interventions and Independence for Elders Study). ${ }^{60}$

\section{Conclusion}

Cognitive impairment is a serious threat to poststroke recovery for patients in all age categories, compromising one's ability to work, and leading to early dependency. A comprehensive neuropsychological evaluation is the best tool for the diagnosis of PSCI. If shorter screening measures are used, it is important to be aware of the fact that their principal range of use is in diagnosing dementia in elderly patients, which may leave less severe conditions undiagnosed. The treatment of PSCI and dementia is based on the effective treatment of vascular risk factors, including lifestyle modification when needed. Treatment of associated conditions such as depression and delirium is important in order to both prevent and manage PSCI and dementia. Early recognition of these conditions is crucial, because when the deficits are serious enough to meet the criteria of dementia, the prognosis becomes deleterious. The key issue to investigate in future research is the role of different risk factors in the transition from PSCI to dementia.

\section{Disclosure}

The authors report no conflicts of interest in this work.

\section{References}

1. O’Brien JT, Erkinjuntti T, Reisberg B, et al. Vascular cognitive impairment. Lancet Neurol. 2003;2(2):89-98.

2. Hachinski V, Iadecola C, Petersen RC, et al. National Institute of Neurological Disorders and Stroke-Canadian Stroke Network vascular cognitive impairment harmonization standards. Stroke. 2006;37(9): 2220-2241.

3. Erkinjuntti T. Vascular cognitive deterioration and stroke. Cerebrovasc Dis. 2007;24 Suppl 1:189-194.

4. Pantoni L, Poggesi A, Inzitari D. Cognitive decline and dementia related to cerebrovascular diseases: some evidence and concepts. Cerebrovasc Dis. 2009;27 Suppl 1:191-196.

5. Vataja R, Pohjasvaara T, Mäntylä R, et al. Depression-executive dysfunction syndrome in stroke patients. Am J Geriatr Psychiatry. 2005;13:99-107.

6. Mok V, Chang C, Wong A, et al. Neuroimaging determinants of cognitive performances in stroke associated with small vessel disease. J Neuroimaging. 2005;15(2):129-137.

7. Tatemichi TK, Paik M, Bagiella E, Desmond DW, Pirro M, Hanzawa LK. Dementia after stroke is a predictor of long-term survival. Stroke. 1994;25(10):1915-1919.

8. Lindén T, Skoog I, Fagerberg B, Steen B, Blomstrand C. Cognitive impairment and dementia 20 months after stroke. Neuroepidemiology. 2004;23(1-2):45-52.

9. van Swieten JC, Koudstaal PJ, Visser MC, Schouten HJ, van Gijn J. Interobserver agreement for the assessment of handicap in stroke patients. Stroke. 1988;19(5):604-607.

10. Kokmen E, Whisnant JP, O'Fallon WM, Chu CP, Beard CM. Dementia after ischemic stroke: a population-based study in Rochester, Minnesota (1960-1984). Neurology. 1996;46(1):154-159.

11. Leys D, Hénon H, Mackowiak-Cordoliani MA, Pasquier F. Poststroke dementia. Lancet Neurol. 2005;4(11):752-759.

12. Barba R, Morin MD, Cemillán C, Delgado C, Domingo J, Del Ser T. Previous and incident dementia as risk factors for mortality in stroke patients. Stroke. 2002;33(8):1993-1998.

13. Hénon H, Durieu I, Lebert F, Pasquier F, Leys D. Influence of prestroke dementia on early and delayed mortality in stroke patients. $J$ Neurol. 2003;250(1):10-16.

14. Pendlebury ST, Rothwell PM. Prevalence, incidence, and factors associated with pre-stroke and post-stroke dementia: a systematic review and meta-analysis. Lancet Neurol. 2009;8(11):1006-1018.

15. Sachdev PS, Brodaty H, Valenzuela MJ, et al. Clinical determinants of dementia and mild cognitive impairment following ischaemic stroke: the Sydney Stroke Study. Dement Geriatr Cogn Disord. 2006;21(5-6): 275-283.

16. Snowdon DA, Greiner LH, Mortimer JA, Riley KP, Greiner PA, Markesbery WR. Brain infarction and the clinical expression of Alzheimer disease. The Nun Study. JAMA. 1997;277(10):813-817.

17. Utter S, Tamboli IY, Walter J, et al. Cerebral small vessel disease-induced apolipoprotein E leakage is associated with Alzheimer disease and the accumulation of amyloid beta-protein in perivascular astrocytes. $J$ Neuropathol Exp Neurol. 2008;67(9):842-856. 
18. Lenzi GL, Altieri M. Short-term evolution as a marker of vascular dementia versus Alzheimer's disease. J Neurol Sci. 2007;257(1-2):182-184.

19. Iadecola C, Park L, Capone C. Threats to the mind: aging, amyloid, and hypertension. Stroke. 2009;40(Suppl 3):S40-S44.

20. Pantoni L, Basile AM, Pracucci G, et al. Impact of age-related cerebral white matter changes on the transition to disability - the LADIS study: rationale, design and methodology. Neuroepidemiology. 2005;24(1-2): 51-62.

21. Inzitari D, Pracucci G, Poggesi A, et al; LADIS Study Group. Changes in white matter as determinant of global functional decline in older independent outpatients: three year follow-up of LADIS (leukoaraiosis and disability) study cohort. BMJ. 2009;339:b2477.

22. Patel M, Coshall C, Rudd AG, Wolfe CD. Natural history of cognitive impairment after stroke and factors associated with its recovery. Clin Rehabil. 2003;17(2):158-166.

23. Rasquin SM, Lodder J, Verhey FR. The effect of different diagnostic criteria on the prevalence and incidence of post-stroke dementia. Neuroepidemiology. 2005;24(4):189-195.

24. Ballard C, Rowan E, Stephens S, Kalaria R, Kenny RA. Prospective follow-up study between 3 and 15 months after stroke: improvements and decline in cognitive function among dementia-free stroke survivors $>75$ years of age. Stroke. 2003;34(10):2440-2444.

25. Oksala NK, Jokinen H, Melkas S, et al. Cognitive impairment predicts poststroke death in long-term follow-up. J Neurol Neurosurg Psychiatry. 2009;80(11):1230-1235.

26. Sibolt G, Curtze S, Melkas S, et al. Poststroke dementia is associated with recurrent ischaemic stroke. $J$ Neurol Neurosurg Psychiatry. 2013;84(7):722-726.

27. Melkas S, Oksala NK, Jokinen H, et al. Poststroke dementia predicts poor survival in long-term follow-up: influence of prestrike cognitive decline and previous stroke. J Neurol Neurosurg Psychiatry. 2009;80(8):865-870.

28. SPS3 Study Group, Benavente OR, Coffey CS, et al. Blood-pressure targets in patients with recent lacunar stroke: the SPS3 randomised trial. Lancet. 2013;382(9891):507-515.

29. Pohjasvaara T, Mäntylä R, Ylikoski R, Kaste M, Erkinjuntti T. Comparison of different clinical criteria (DSM-III, ADDTC, ICD-10, NINDS-AIREN, DSM-IV) for the diagnosis of vascular dementia. National Institute of Neurological Disorders and Stroke-Association Internationale pour la Recherche et l'Enseignement en Neurosciences. Stroke. 2000;31(12):2952-2957.

30. Hauw JJ, De Girolami U, Zekry D. The neuropathology of vascular and mixed dementia and vascular cognitive impairment. Handb Clin Neurol. 2008;89:687-703.

31. Hodkinson HM. Evaluation of a mental test score for assessment of mental impairment in the elderly. Age Aging. 1972;1(4):233-238.

32. Jokinen H, Kalska H, Mäntylä R, et al. Cognitive profile of subcortical ischaemic vascular disease. J Neurol Neurosurg Psychiatry. 2006;77(1):28-33.

33. Douiri A, Rudd AG, Wolfe CD. Prevalence of poststroke cognitive impairment: South London Stroke Register 1995-2010. Stroke. 2013;44(1):138-145.

34. Makin SD, Turpin S, Dennis MS, Wardlaw JM. Cognitive impairment after lacunar stroke: systematic review and meta-analysis of incidence, prevalence and comparison with other stroke subtypes. $J$ Neurol Neurosurg Psychiatry. 2013;84(8):893-900.

35. Jokinen H, Gouw AA, Madureira S, et al; LADIS Study Group. Incident lacunes influence cognitive decline: the LADIS study. Neurology. 2011;76(22):1872-1878.

36. Edwards JD, Jacova C, Sepehry AA, Pratt B, Benavente OR. A quantitative systematic review of domain-specific cognitive impairment in lacunar stroke. Neurology. 2013;80(3):315-322.

37. Gold G, Kövari E, Herrmann FR, et al. Cognitive consequences of thalamic, basal ganglia, and deep white matter lacunes in brain aging and dementia. Stroke. 2005;36(6):1184-1188.
38. Bour A, Rasquin S, Boreas A, Limburg M, Verhey F. How predictive is the MMSE for cognitive performance after stroke? J Neurol. 2010; 257(4):630-637.

39. Nys GM, van Zandvoort MJ, de Kort PL, Jansen BP, Kappelle LJ, de Haan EH. Restrictions of the Mini-Mental State Examination in acute stroke. Arch Clin Neuropsychol. 2005;20(5):623-629.

40. Pendlebury ST, Cuthbertson FC, Welch SJ, Mehta Z, Rothwell PM. Underestimation of cognitive impairment by Mini-Mental State Examination versus the Montreal Cognitive Assessment in patients with transient ischemic attack and stroke: a population-based study. Stroke. 2010;41(6):1290-1293.

41. Salvadori E, Pasi M, Poggesi A, Chiti G, Inzitari D, Pantoni L. Predictive value of MoCA in the acute phase of stroke on the diagnosis of midterm cognitive impairment. J Neurol. 2013;260(9):2220-2227.

42. de Koning I, van Kooten F, Koudstaal PJ, Dippel DW. Diagnostic value of the Rotterdam-CAMCOG in post-stroke dementia. J Neurol Neurosurg Psychiatry. 2005;76(2):263-265.

43. Restaino M, Matthews FE, Minett T, Albanese E, Brayne C, Stephan BC. Predicting risk of 2-year incident dementia using the CAMCOG total and subscale scores. Age Ageing. 2013;42(5):649-653.

44. Ferris SH. General measures of cognition. Int Psychogeriatr. 2003; 15 Suppl 1:215-217.

45. Ylikoski R, Jokinen H, Andersen P, et al; LADIS Study Group. Comparison of the Alzheimer's Disease Assessment Scale Cognitive Subscale and the Vascular Dementia Assessment Scale in differentiating elderly individuals with different degrees of white matter changes. The LADIS Study. Dement Geriatr Cogn Disord. 2007;24(2):73-81.

46. Lees R, Corbet S, Johnston C, Moffitt E, Shaw G, Quinn TJ. Test accuracy of short screening tests for diagnosis of delirium or cognitive impairment in an acute stroke unit setting. Stroke. 2013;44(11): 3078-3083.

47. The 4AT test group [webpage on the Internet]. Edinburgh: The 4A Test: screening instrument for cognitive impairment and delirium. 2011. Available from: http://www.the4at.com/the-4at. Accessed May 1, 2013.

48. Dufouil C, Chalmers J, Coskun O, et al; PROGRESS MRI Substudy Investigators. Effects of blood pressure lowering on cerebral white matter hyperintensities in patients with stroke: the PROGRESS (Perindopril Protection Against Recurrent Stroke Study) Magnetic Resonance Imaging Substudy. Circulation. 2005;112(11):1644-1650.

49. Schiffrin EL. Blood pressure lowering in PROGRESS (Perindopril Protection Against Recurrent Stroke Study) and white matter hyperintensities: should this progress matter to patients? Circulation. 2005;112(11):1525-1526.

50. Gorelick PB, Scuteri A, Black SE, et al; American Heart Association Stroke Council, Council on Epidemiology and Prevention, Council on Cardiovascular Nursing, Council on Cardiovascular Radiology and Intervention, and Council on Cardiovascular Surgery and Anesthesia. Vascular contributions to cognitive impairment and dementia: a statement for healthcare professionals from the american heart association/ american stroke association. Stroke. 2011;42(9):2672-2713.

51. Malouf R, Birks J. Donepezil for vascular cognitive impairment. Cochrane Database Syst Rev. 2004;(1):CD004395.

52. Birks J, Craig D. Galantamine for vascular cognitive impairment. Cochrane Database Syst Rev. 2013;4:CD004746.

53. McShane R, Areosa Sastre A, Minakaran N. Memantine for dementia. Cochrane Database Syst Rev. 2006;(2):CD003154.

54. Erkinjuntti T, Kurz A, Gauthier S, Bullock R, Lilienfeld S, Damaraju CV. Efficacy of galantamine in probable vascular dementia and Alzheimer's disease combined with cerebrovascular disease: a randomised trial. Lancet. 2002;359(9314):1283-1290.

55. Chollet F, Tardy J, Albucher JF, et al. Fluoxetine for motor recovery after acute ischaemic stroke (FLAME): a randomised placebo-controlled trial. Lancet Neurol. 2011;10(2):123-130.

56. Royall DR, Cordes JA, Román G, et al. Sertraline improves executive function in patients with vascular cognitive impairment. J Neuropsychiatry Clin Neurosci. 2009;21(4):445-454. 
57. Alexopoulos GS, Kiosses DN, Klimstra S, Kalayam B, Bruce ML. Clinical presentation of the "depression-executive dysfunction syndrome" of late life. Am J Geriatr Psychiatry. 2002;10(1):98-106.

58. Melkas S, Laurila JV, Vataja R, et al. Post-stroke delirium in relation to dementia and long-term mortality. Int J Geriatr Psychiatry. 2012;27(4): 401-408.

59. Boecker H. On the emerging role of neuroimaging in determining functional and structural brain integrity induced by physical exercise: impact for predictive, preventive, and personalized medicine. EPMA J. 2011;2(3):277-285.
60. Fielding RA, Rejeski WJ, Blair S, et al; LIFE Research Group. The Lifestyle Interventions and Independence for Elders Study: design and methods. J Gerontol A Biol Sci Med Sci. 2011;66(11):1226-1237.

\section{Publish your work in this journal}

Degenerative Neurological and Neuromuscular Disease is an international, peer-reviewed, open access journal focusing on research into degenerative neurological and neuromuscular disease, identification of therapeutic targets and the optimal use of preventative and integrated treatment interventions to achieve improved outcomes, enhanced survival and quality of life for the patient. The manuscript management system is completely online and includes a very quick and fair peer-review system. Visit http://www.dovepress.com/testimonials.php to read real quotes from published authors.

Submit your manuscript here: http://www.dovepress.com/degenerative-neurological-and-neuromuscular-disease-journal 